\title{
Abstracts of Theses Approved for the MSc Degree at the Faculty of Medicine, Health Sciences Centre, Kuwait University
}

\section{1 \\ Trichosporon asahii: Studies on Antifungal Susceptibility and Molecular Characterization M. Al-Mahmeed}

Department of Microbiology, Faculty of Medicine, Kuwait University

Objective: Trichosporon asahii is the major cause of deep-seated trichosporonosis and is an emerging pathogen of granulocytopenic patients. This study investigated the antifungal susceptibility profile, genetic diversity and species-specific identification of isolates of Trichosporon species recovered from various clinical specimens in Kuwait. Materials and Methods: Twenty-nine clinical isolates of Trichosporon species initially identified as $T$. asahii by the Vitek 2 system were studied for colony and microscopic characteristics. The molecular identification of Trichosporon species was performed by PCR amplification of the internally transcribed spacer (ITS) regions 1 and 2 of rDNA with species-specific or panfungal primers and direct DNA sequencing of the amplicons. The genotypic diversity of the isolates was studied by randomly amplified polymorphic DNA (RAPD) with two RAPD primers. Minimum inhibitory concentrations (MICs) of amphotericin B, 5-flucytosine, fluconazole, itraconazole, ketoconazole, and voriconazole were determined by the NCCLS broth microdilution (BMD) method and E-test. Results: Of the 2 morphotypes recognized, 25 isolates exhibited cream-colored, rugose colonies with a farinose covering while the other 4 isolates appeared more folded and verrucous. Both morphotypes were indistinguishable in microscopic features and formed similar hyphae and abundant arthroconidia. The amplification of rDNA using $T$. asahii-specific primers yielded positive results from only 25 of the 29 isolates and their identity was further confirmed by DNA sequencing of the ITS-1 and ITS-2 regions. The identity of the remaining 4 isolates was deduced by
PCR amplification of rDNA using panfungal primers followed by direct DNA sequencing of the ITS- 1 and ITS- 2 regions contained in the amplified products. These studies established the identity of the latter 4 isolates as $T$. asteroides. The fingerprinting of strains of Trichosporon species carried out with two RAPD primers, GAC-1 and M13, showed limited heterogeneity among the 25 T. asahii and $4 \mathrm{~T}$. asteroides isolates. The $\mathrm{MIC}_{90}$ for voriconazole, fluconazole, ketoconazole, itraconazole, amphotericin B and flucytosine were $0.125,4,1,0.5,4$ and $>32 \mu \mathrm{g} / \mathrm{ml}$ by the BMD test, and 0.094, 8, $0.75,1,4$ and $>32 \mu \mathrm{g} / \mathrm{ml}$ by the E-test, respectively. A notable observation was that the E-test showed lower MICs for amphotericin B than the BMD reference method. Sixty-eight percent of T. asahii isolates were inhibited at $\leq 1 \mu \mathrm{g} / \mathrm{ml}$ by the E-test as against only $36 \%$ by the BMD method. Moreover, E-test MICs for amphotericin $\mathrm{B}$ were distributed over a wider range than BMD MICs. Time-kill study carried out with $2 \mathrm{~T}$. asahii isolates revealed that the fluconazole possessed only fungistatic activity against this species. Antifungal susceptibility profiles of the $4 T$. asteroides isolates by the E-test and BMD method against the 6 antifungal agents were comparable to T. asahii isolates. Voriconazole exhibited highest in vitro activity, followed by fluconazole, itraconazole, ketoconazole and amphotericin B. Conclusions: The study demonstrated that T. asahii is the most prevalent Trichosporon species in clinical specimens in Kuwait. Apart from the morphological and biochemical characteristics, molecular characterization is needed for speciesspecific identification of the isolates. No morphotypic or fingerprinting patterns were identified among the Trichosporon species isolated from similar clinical specimens or anatomic sites. Among the azole antifungal agents, voriconazole exhibited excellent activity against Trichosporon species followed by fluconazole.

Z.U. Khan (supervisor)

S. Ahmad (co-supervisor)

\section{KARGER}

Fax +4161306 1234 E-Mail karger@karger.ch www.karger.com
C 2005 S. Karger AG, Basel

1011-7571/05/0145-0370\$22.00/0

Accessible online at:

www.karger.com/mpp 
2

\section{Establishment of a Molecular Assay to Detect the Mumps Virus Genome in Clinical Specimens and Study the Immune Status of Children in Kuwait to Mumps Virus}

\author{
Ola Hassan Moghnai \\ Department of Microbiology, Faculty of Medicine, \\ Kuwait University
}

Despite the extensive worldwide mumps vaccination programs, mumps outbreaks still occur and a number of vaccine-related aseptic meningitis (AM) cases have also been documented. Years after the introduction of the rigorous vaccination campaign against measles-mumps-rubella (MMR) here in Kuwait, mumps and AM cases have been reported every year. The occurrence of mumps outbreaks during the postvaccination era may be explained by three factors: (1) the use of different attenuated mumps virus $(\mathrm{MuV})$ strains in the vaccine; (2) different antigenic properties of circulating wild mumps strains, and (3) low level of immunity. Though enteroviruses are considered to be the leading cause of $\mathrm{AM}, \mathrm{MuV}$ is responsible for a proportion of the AM cases. This study aimed at: (1) determining the prevalence of MuV-specific antibodies; (2) investigating the level of protective immunity to $\mathrm{MuV}$, and (3) establishing a sensitive molecular method for diagnosing MuV-related meningitis cases. The effectiveness of the mumps vaccination campaign in Kuwait was studied by determining the prevalence of $\mathrm{MuV}$-specific antibodies using enzyme-linked immunosorbent assay, while the protective immunity was investigated by the virus neutralization test established in the laboratory. For diagnosing mumps meningitis, a molecular method, reverse transcriptasepolymerase chain reaction (RT-PCR), has been established and used for the detection of the $\mathrm{MuV}$ genome in cerebrospinal fluid (CSF) samples. The prevalence of $\mathrm{MuV}$-specific antibodies after two doses of MMR vaccine reached $92 \%$ by the age of 5-12 years. The protective, neutralizing antibodies could be detected in sera of $94 \%$ of children aged 11-16 years. In the younger group (510 years), the protective antibodies could be detected more frequently to the homologous (94\%) than to the heterologous (77\%) $\mathrm{MuV}$ strain. The two-dose MMR vaccination schedule implemented in Kuwait in 1994 resulted in a high prevalence rate of antibodies to homologous and heterologous $\mathrm{MuV}$ strains and revealed the possibility that antigenically different $\mathrm{MuV}$ genotypes may be present in Kuwait. Using RT-PCR, which targeted the nucleoprotein region, the $\mathrm{MuV}$ genome could be detected in $6.2 \%$ of CSF samples tested. The established molecular method proved to be sensitive and specific for the detection of the $\mathrm{MuV}$ genome and it could be used to diagnose $\mathrm{MuV}$-associated AM cases.

Dr. Alexander Pacsa (supervisor)

Dr. Gyorgy Szucs (co-supervisor)

\section{3}

Development of a Murine Model for Studying the Effect of Splenectomy on the Outcome of Lower Respiratory Tract Infection with Streptococcus pneumoniae

Suha AbdullGhafour Al-AbdullGhafour

Department of Microbiology, Faculty of Medicine, Kuwait University

Streptococcus pneumoniae (pneumococcus) is a common commensal of the upper respiratory tract epithelium of healthy children and adults and a common agent in a wide variety of infections. Immunological deficiencies predispose to pneumococcal infection. The spleen is an important organ of the immune system and splenectomy has been shown to lead to infections with encapsulated bacteria. $S$. pneumoniae accounts for the majority of bacterial infections in these patients. The main aim of this project was to develop a murine model for studying the pathogenicity of $S$. pneumoniae and to use this model to ascertain the effect of splenectomy on the outcome of respiratory tract infection. Human $S$. pneumoniae isolates were characterized and serially passaged in Balb/c mice to select virulent strains. Respiratory infection was induced by inoculating Balb/c and C57BL mice intranasally with $50 \mu \mathrm{l}$ of $S$. pneumoniae suspension from the most virulent strain after passaging. The development of pneumonia was determined by clinical symptoms, level of bacteremia and histological and microbiological examination of the lungs. Profound respiratory tract infection was produced by a high inoculum dose $\left(10^{9} \mathrm{CFU} / \mathrm{ml}\right)$ in both strains but not at lower doses. Differences in the susceptibility of Balb/c and C57BL mice to S. pneumoniae infection were found. Contrary to expectations, no major differences were found in the level of bacteremia and in the lung pathology in splenectomized versus nonsplenectomized Balb/c mice.

Prof. C. H. J. Ford (supervisor)

Prof. V. O. Rotimi (co-supervisor)

\section{4 \\ Modulation of Type 1 Cytokine Responses in Pregnancy}

\section{Esraa Mubarak Al-Mutawa}

Department of Microbiology, Faculty of Medicine, Kuwait University

Introduction and Objective: Pregnancy loss is a problem of great medical, scientific, social and economic relevance all over the world. A complete understanding of the causative factors of pregnancy loss would help us devise effective methods for its prevention and treatment. Recent research suggests the possible involvement of cell-mediated immunity and cytokines in recurrent spontaneous abortion (RSA) and preterm labor (PTL), two of the most common complications of pregnancy. Th1-type or type 1 cytokines are injurious to the conceptus and maternal type 1 reactivity is generally harmful to murine pregnancy. Women with a history of unexplained RSA and women with PTL evince a bias towards type 1 reactivity. Successful pregnancy, on the other hand, is biased towards 
dominance of maternal type 2 or Th2-type reactivity; a strong bias towards type 2 maternal reactivity has been demonstrated in normal pregnancy. If strong maternal type 1 immunity is indeed responsible for pregnancy loss, we may speculate that the suppression or modulation of type 1 cytokines and thus the 'redirection' of maternal reactivity away from type 1 predominance may serve to prevent cytokine-mediated pregnancy loss. There are suggestions that progesterone may facilitate this modulation of type 1 responses. Progesterone has been shown to favor the development of type 2 responses in vitro and has been shown to reduce the production of type 1 cytokines by lymphocytes from women with a history of RSA. The studies described in the thesis were undertaken with a view to ascertain whether dydrogesterone (Duphaston), an orally administered progesterone derivative, and progesterone-induced blocking factor (PIBF) can modulate human cytokine responses. Materials and Methodology: Peripheral blood mononuclear cells isolated from blood samples obtained from women with unexplained RSA and from women undergoing PTL were stimulated with a mitogen in the presence and absence of progesterone, Duphaston and PIBF. Levels of type 1 and type 2 cytokines were estimated by a sensitive ELISA. Results: The results indicate that Duphaston and PIBF do indeed bring about a type $1 \rightarrow$ type 2 cytokine shift, suggesting that modulation of maternal cytokine profiles and cellular reactivity is possible with Duphaston and PIBF. This effect of Duphaston can be inhibited using RU486 (a progesterone antagonist), indicating that Duphaston mediates cytokine-modulating effects via the progesterone receptor. Conclusion: The results emerging from this work indicate the potential merit of these molecules in therapeutic intervention in pregnancy loss, based on their ability to effect a shift from the potentially harmful maternal type 1 reactivity to a more pregnancy-conducive type 2 cytokine profile.

Prof. Raj Raghupathy (supervisor)

Dr. Raja'a Al-Attiyah (co-supervisor)

5

\section{9mTc Pertechnetate Thyroid Uptake: Reliability of the Test}

Nafisa Abdullah H. Kazem

Department of Nuclear Medicine, Faculty of Medicine, Kuwait University

Introduction: ${ }^{99 \mathrm{~m}} \mathrm{Tc}$ pertechnetate thyroid scintigraphy is a valuable procedure in many clinical situations. However, if a thyroid uptake is required, non-utilization of uptake determination by quantitative methods for this test necessitates performing a radioiodine uptake measurement. The aims of this study were: to establish the range of ${ }^{99 \mathrm{~m}} \mathrm{Tc}$ pertechnetate thyroid uptake (TcTU) in euthyroid subjects; to study the correlation between TcTU and ${ }^{131} \mathrm{I}$ thyroid uptake; to derive a conversion formula from TcTU to 24hour iodine uptake and to study the relationship between TcTU, clinical parameters and thyroid hormone levels. Materials and Methods: This is a prospective consecutive case study conducted over a period of 1 year where patients referred for both thyroid uptake and scintigraphy were included. TcTU was derived from 20-min thyroid image performed using ${ }^{99 \mathrm{~m}} \mathrm{Tc}$ pertechnetate and a gamma camera equipped with a pinhole collimator. ${ }^{131}$ I uptake was measured using a counting probe $24 \mathrm{~h}$ after oral administration of ${ }^{131}$ I. Results and Discussion: 229 subjects (47 males, 182 females) were studied. Among 79 euthyroid patients, TcTU was $1.7 \pm 1.4$. In the hyperthyroid and subclinical hyperthyroid groups, mean TcTU was $11.8 \pm 9.6$ and $2.4 \pm 3.1$, respectively. A highly significant positive correlation was found between TcTU and ${ }^{131} \mathrm{I}$ uptake $(\mathrm{r}=0.837, \mathrm{p}<0.001)$. Both studies showed differences between the euthyroid and hyperthyroid groups and between the frank hyperthyroid and subclinical hyperthyroid groups. Both parameters showed significant correlations with some of the clinical parameters, thyroid function tests, and the size of the thyroid gland. TcTU, however, in many of these comparisons had more significant and stronger associations than the 24-hour ${ }^{131} \mathrm{I}$ uptake. A conversion equation was derived $\left({ }^{131}\right.$ I uptake $=14.6 \mathrm{In}$ [TcTU] +26.9$)$ with a correlation coefficient $\mathrm{R}^{2}$ of 0.67 . Conclusion: TcTU is an additional reliable quantitative parameter which potentially eliminates the need for separate ${ }^{131}$ I uptake measurements in patients with thyroid disorders.

\section{Dr. Ghulam M. Shah Syed (supervisor) \\ Dr. Henry W. Fielding (co-supervisor)}

\section{6 \\ A Comparison of Myocardial Perfusion Gated SPECT Studies Obtained at Rest and after Stress}

Mahmoud A. Al-Feeli

Department of Nuclear Medicine, Faculty of Medicine, Kuwait University

Objective: To compare post-stress and rest gated SPECT myocardial perfusion imaging in patients with ischemic heart disease in order to assess the post-stress stunning and the variables causing the phenomenon. Subjects and Methods: This is a prospective study of 318 consecutive adult patients undergoing stress and rest gated SPECT using a two-day ${ }^{99 \mathrm{~m}}$ Tc tetrofosmin protocol. Bruce II protocol treadmill stress $(n=93)$ or intravenous dipyridamole pharmacologic stress $(n=225)$ was used for stressing. Ejection fractions and left ventricular (LV) end-diastolic and end-systolic volumes (EF, EDV and ESV, respectively) were calculated using the Cedar Sinai's Quantitative Gated SPECT software. Perfusion defects were scored using a 20-segment scoring system. Results: Statistical analysis, performed by applying paired t test, showed significant differences between post-stress and rest EF and ESV for total patient population. Analysis based on the type of stress showed significantly lower EF and significantly higher ESV on post-stress studies for the dipyridamole group (all $p$ values $<0.01$ ) Multiregression analysis using differences in post-stress and rest EF, EDV and ESV as dependent and summed stress scores (SSS), type of stress, delay time, previous myocardial infarction and LV size as independent variables showed statistically significant associations between higher SSS (>13), LV size on post-stress for EDV and ESV differences. Higher volumes were noted on post-stress studies. Conclusion: A subgroup of patients who have significant ischemia show stunning as manifested by high EDV and ESV differences and dilated LV on post-stress studies.

Dr. Ghulam M. Shah Syed (supervisor)

Dr. Henry W. Fielding (co-supervisor) 
7

Single Photon Emission Computed Tomography of Regional Cerebral Blood Flow in Patients with Sickle Cell Disease

Fareeda Ahmed Al-Kandari

Department of Nuclear Medicine, Faculty of Medicine, Kuwait University

Objective: To use SPECT to study brain perfusion in patients with sickle cell disease (SCD) after administration of technetium$99 \mathrm{~m}$ hexamethylpropylene amine oxime ( $\left.{ }^{99 \mathrm{~m}} \mathrm{Tc}-\mathrm{HMPAO}\right)$, and correlate the findings with those of demography, physical examination, MRI and haematological profile. Subjects and Methods: The study involved 21 patients (12 male, 9 female) known to have had SCD for at least 5 years. The patients were not in acute crisis, had normal neurological assessment, no known history of stroke or transient ischaemic episode or previous abnormal CT or MRI brain scan, and were right-handed. Demographic data and haematological profile were obtained from patients' medical records. Brain SPECT was performed after intravenous injection of $740 \mathrm{MBq}$ $(20 \mathrm{mCi})^{99 \mathrm{~m}} \mathrm{Tc}-\mathrm{HMPAO}$ in adult or an appropriate dose in paediatric patients. The scans were visually interpreted by two nuclear medicine consultants who reached a decision by consensus. Observed brain perfusion deficits (BPD) were graded as normal, mild, moderate and severe. MRI of 19 patients was done within 3 months of brain SPECT study and interpreted by a consultant radiologist. Results: Of the 21 patients, 7 had BPD, 4 were moderate to severe and the remaining 3 were mild. The BPD were mostly either in the frontal lobe or in combination with the temporal and/or parietal lobe. MRI was abnormal in 2 patients. The BPD were not statistically correlated with patients' demographic data, haematological profiles and MRI with a $p$ value of $>0.05$. Conclusion: SPECT imaging was successfully used to detect early BPD. Such early detection may be clinically useful in the subsequent follow-up of such patients, since it is known that cerebral perfusion deficit could lead to silent infarct and/or to overt stroke and affect cognitive skills.

Prof. Azuwuike Owunwanne (supervisor)

Dr. Ghulam Mustafa Shah Syed (co-supervisor)

\section{8 \\ Correlation of Loss of Heterozygosity on Chromosomes 4 and 8p with Disease-Free Survival in Early Colorectal Carcinoma}

\section{A.Y.H. Al-Hakim}

Department of Pathology, Faculty of Medicine, Kuwait

University, Kuwait

Introduction and Objective: The identification of salient molecular markers revealing the metastatic destiny of a primary colorectal tumor and the detection of micrometastases as early as possible in the patient are paramount objectives in colorectal carcinoma (CRC) research. Loss of heterozygosity $(\mathrm{LOH})$ is a very common chromosomal aberration in CRC, and its occurrence is indisputably linked to the loss of both tumor suppressor genes and metastasis suppressor genes (MSG). The discovery and mapping of
MSG whose loss unequivocally plays a definitive role in the metastatic progression of CRC is of immense significance in predicting disease-free survival. This study aims to correlate LOH and loss of putative MSG on chromosomes 4 and $8 \mathrm{p}$ with decreased diseasefree survival in Dukes' stages A and B of early CRC. Materials and Methods: DNA extracted from 78 blocks of archival paraffin-embedded paired normal and tumor CRC tissue was subjected to allelotyping with nine fluoroscently labeled polymorphic microsatellite primers for each of chromosomes 4 and $8 \mathrm{p}$. Allelic loss was subsequently assessed for each microsatellite locus and tested for association with disease-free survival using Kaplan-Meier survival curves. Results: No association was detected between LOH and disease-free survival at any of the nine microsatellite markers on chromosome 8 p. LOH at 2 of the 9 markers investigated on chromosome 4 revealed a significant association with progression to metastatic disease and decreased disease-free survival. Conclusion: Correlation of $\mathrm{LOH}$ at these two loci with decreased diseasefree survival provides evidence for the presence of a putative MSG on chromosome 4 whose loss is highly associated with recurrent metastatic disease in Dukes' A and B CRC patients. LOH at these two loci should be used as markers of occult micrometastases in early CRC. Further investigation is required to explore this region and others on chromosome 4 that may harbor unmapped MSG involved in the mestastatic progression of CRC.

F. Al-Mulla (supervisor)

S.O. Olusi (co-supervisor)

\section{9 \\ Effect of Angiotensin-I-Converting Enzyme Gene Polymorphism on the Plasma Angiotensin-I-Converting Enzyme Concentration and the Development of Microalbuminuria in Type 2 Diabetic Patients of Kuwait}

Doha S. Al-Khrayef

Department of Pathology, Faculty of Medicine, Kuwait University

Background: Diabetes mellitus is a common cause of morbidity and mortality in Kuwait. Diabetic nephropathy is a complication of diabetes that develops in close to $30 \%$ of diabetic patients and is the leading cause of end-stage renal failure. Genetic factors play an important role in the development of diabetic nephropathy and the insertion/deletion (I/D) polymorphism of the angiotensinI-converting enzyme (ACE I) gene has been found to be associated with nephropathy in type 2 diabetic patients. Objectives: To determine whether (a) plasma ACE I concentration is under the control of gene polymorphism and (b) an association exists between the ACE I I/D polymorphism and microalbuminuria in Kuwaiti type 2 diabetic patients. Method: 125 Kuwaiti type 2 diabetic patients and 50 sex- and age-matched control subjects were studied. Nephropathy status was determined according to the urinary albumin:creatinine ratio. The ACE I concentration was determined by ELISA; the I/D polymorphism was determined by PCR. Results: Plasma ACE I concentration was genetically influenced and patients with the DD genotype have the highest plasma concentration, 
followed by those with ID and II genotypes. The percentages of ACE I genotypes (DD, ID, II) in normoalbuminuric patients were $17.6,44.2,38.2$, respectively, while in microalbuminuric patients they were $87.8,10.2,2.0$, respectively, and in macroalbuminuric patients they were 100, 0, 0, respectively. Conclusion: This study showed that the circulating ACE I concentration is under control of gene polymorphism. Also, this study showed a positive association between the $\mathrm{D}$ allele and microalbuminuria in Kuwaiti type 2 diabetic patients.

O. A. Mojiminiyi (supervisor)

F. Al-Mulla (co-supervisor)

N. Abdella (co-supervisor)

\section{0 \\ BRCA1 Gene Expression in Breast Cancer in Kuwait}

Mahera Abdlrahman Amirrad

Department of Pathology, Faculty of Medicine,

Kuwait University

Introduction and Objective: Breast cancer is the third most common form of cancer worldwide after lung and stomach cancer, and it is the most common form among women. Epidemiological studies in the Kuwaiti population also show that breast cancer is the most common cancer in Kuwaiti women. The strongest known epidemiological risk factor for developing breast cancer is a family history of the disease. It is estimated that about $5-10 \%$ of breast cancer cases may be due to inherited predisposition, and recent studies show that BRCA1 gene plays a critical role in both familial and sporadic types of breast cancer. In the absence of reliable data on familial breast cancer in Kuwait, a study of $B R C A 1$ gene expression either at the mRNA level or at the protein level should assist in defining a subgroup that would contain familial forms of disease. Methods: In the present study, 48 random samples of archival paraffin-embedded breast cancer tissues from the Mubarak Al-Kabeer hospital were studied for $B R C A 1$ gene expression. BRCA1 protein expression was studied by an immunohistochemical method utilizing antibodies against different epitopes on BRCA1 protein. A molecular technique, reverse transcription-polymerase chain reaction, was used to detect $B R C A 1$ expression at the mRNA level. Results: Significant lack of expression of BRCA1 mRNA (79\%) and protein $(83 \%)$ was found in the majority of breast cancer tissues studied. As in previous studies, an inverse relationship was demonstrated between BRCA1 (mRNA and protein) expression and histological grade of breast cancer. Also, negative BRCA1 (mRNA and protein) expression correlated with negative estrogen and progesterone receptor status of the breast cancer tissues, but with overexpression of c-erbB2. Conclusion: These findings confirm the inverse relationship between $B R C A 1$ expression and parameters that determine poor prognosis in breast cancer. The breast cancer patients who had no BRCA1 expression (both mRNA and protein) may be further investigated to ascertain a possible genetic basis for their cancer. Segregating hereditary breast cancer patients in Kuwait will be of special help for the family members of such patients, who can have the opportunity of $B R C A 1$ screening tests and the advantage of prophylactic management. The study is also important in estab- lishing, for the first time in Kuwait, a method of detecting BRCA1 mRNA expression in archival material and opens the way for future studies into the biology of breast cancer in the region.
J. T. Anim (supervisor)
F. Al-Mulla (co-supervisor)

\section{1 \\ Introduction of Genomic Aberrations and Their Expressions in a Colorectal Cancer Cell Line (Colo320)}

\section{R.A. Al-Tamimi \\ Department of Pathology, Faculty of Medicine, Kuwait University}

Introduction and Objectives: Genetic instability is an evident common hallmark of later aggressive stages of carcinogenesis. Cancer-related genetic instability involves gross increases in DNA content per nucleus through multiple amplifications of specific regions (amplicons), and/or increased copy numbers (aneuploidy) of chromosomes, along with imbalanced deletions. The majority of published research agrees that all genomic amplifications are overexpressed and deletions suppressed; however, a few publications disagree and suggest variable expression levels of these genomic amplifications. We aim to clarify this discrepancy by assaying genomic and expression profiles of a colorectal cancer cell line. Materials and Methods: Colo320 cell line was optimally cultured and cells were prepared for genomic DNA extraction and total RNA extraction. A normal human metaphase spread was prepared for comparative genomic hybridization $(\mathrm{CGH})$ and biotin-labeled normal colon and Colo320 DNAs were hybridized to it. Total DNAs and RNAs of normal colon and Colo320 were used to generate differentially labeled fluorescent cDNA probes to be hybridized to $16 \mathrm{~K}$ human cDNA array and 7.6K expression oligonucleotide microarray slides, respectively. Real-time PCR was used to confirm expression microarray results. Results: CGH images displayed amplifications and deletions in various chromosomal regions; furthermore, the $\mathrm{CGH}$ array profile detected many single gene amplifications and deletions. Genes of various functions within the $\mathrm{CGH}$ amplified regions were assayed for their expression using oligonucleotide microarrays. Versatile expression levels were shown for genes with unstable copy numbers as detected by CGH and CGH array. No significant correlation $(\mathrm{p}=0.168)$ was found between gene copy number and its corresponding expression. Conclusions: Genomic amplifications are not necessarily overexpressed and there is selectivity in the genes to be activated for expression, overexpression and suppression. This finding implicates multiple mechanisms in the regulation of gene expression during cancer progression. The majority of genes that showed altered genomic and/or expressive profiles were of growth regulation, signal transduction and gene transcription functions.

F. Al-Mulla (supervisor) 


\section{2 \\ Attenuated Vascular Reactivity to Vasopressin in Pregnancy: Role of Heme Oxygenase and Rho-Kinase}

\section{Maram Gamal Katoue}

Department of Pharmacology and Toxicology,

Faculty of Medicine, Kuwait University

Pregnancy is associated with attenuated vascular responsiveness to vasoconstrictor agents. The mechanism(s) responsible for the attenuated responsiveness is/are not yet defined. In this study, the possible roles of heme oxygenase $(\mathrm{HO}) / \mathrm{CO}$ and RhoA/Rho-kinase pathways in mediating the decreased vascular reactivity in pregnancy were evaluated in thoracic aorta segments from pregnant and age-matched nonpregnant rats. Vascular reactivity to arginine vasopressin (AVP) was reduced in pregnancy. This decrease affected maximum response, but not sensitivity. The results indicate that increased nitric oxide (NO) release cannot explain the decreased vascular reactivity to AVP during pregnancy. Western immunoblotting revealed that $\mathrm{HO}-2$ but not $\mathrm{HO}-1$ was expressed in the aorta from pregnant and nonpregnant rats. Even though the expression of HO-2 was increased in pregnancy, inhibition of $\mathrm{HO}$ activity did not reverse the depressed reactivity to AVP, indicating that it is not due to CO. Vasopressin-induced contraction utilizes $\mathrm{Ca}^{2+}$ from both extracellular and intracellular sources. Calcium channel blockade was more effective in suppressing the response to AVP in nonpregnant rats suggesting a functional alteration of the voltage-operated calcium channels during pregnancy. ROCK-I and ROCK-II were expressed in the rat aorta and the level of expression was decreased in pregnancy. Inhibition of Rho-kinase activity was less effective in pregnant rats compared to nonpregnant rats. The present results do not support a role for $\mathrm{NO}$ or $\mathrm{CO}$ in mediating the decreased vascular reactivity in pregnancy. The results, however, suggest that pregnancy affects $\mathrm{Ca}^{2+}$ homeostasis by inhibiting $\mathrm{Ca}^{2+}$ mobilization and agonist-enhanced $\mathrm{Ca}^{2+}$ sensitivity of the myofilaments.

Prof. Mabayoje A. Oriowo (supervisor)

Dr. Islam Khan (co-supervisor)

\section{3 \\ Role of TNF- $\alpha$ in Myocardial Reperfusion Injury: Protective Efficacy of Hyperthermic \\ Lamia Jawdat Hoteit \\ Department of Physiology, Faculty of Medicine, Kuwait University} Preconditioning and $17 \beta$-Estradiol Treatment

Introduction: Relatively little is known about the protective effect of either ischemic preconditioning or $17 \beta$-estradiol against the direct myocardial effects of tumor necrosis factor- $\alpha$ (TNF- $\alpha)$ that contributes to myocardial ischemia/reperfusion injury (I/R). Objectives: (1) To evaluate the effects of exogenous and endogenous TNF- $\alpha$ on myocardial functions. (2) To compare the cardioprotective actions of hyperthermic ischemic preconditioning and of $17 \beta$-estradiol after TNF- $\alpha$ perfusion and I/R. Methods: The isolated aorta-perfused rat heart model (Landendorff) was used. For the TNF- $\alpha$ perfusion studies, male rat hearts were perfused for 60 min with TNF- $\alpha$ containing buffer, using the following protocols ( $\mathrm{n}=5$ for each protocol): (a) TNF- $\alpha(20 \mathrm{ng} / \mathrm{ml}$ perfusate) perfusion for $60 \mathrm{~min}$; (b) normothermic $\left(37^{\circ} \mathrm{C}\right)$ and (c) and (d) hyperthermic ( 40 or $42^{\circ} \mathrm{C}$ ) ischemic preconditioned hearts; (e) normal or (f) preconditioned hearts derived from $17 \beta$-estradiol $(250 \mu \mathrm{g} / \mathrm{kg}$ i.m.)pretreated rats; (g) normal or (h) preconditioned hearts perfused for $60 \mathrm{~min}$ with TNF- $\alpha$ and $17 \beta$-estradiol $[0.7 \mathrm{ng} / \mathrm{ml}$ of coronary flow $(\mathrm{CF}) \times \mathrm{kg}$ body weight] containing buffer. For the I/R studies, male rat hearts were used according to the following protocols $(\mathrm{n}=$ 5 for each): (a) ischemia $\left(37^{\circ} \mathrm{C}\right)$ for $40 \mathrm{~min}$; (b) normothermic $\left(37^{\circ} \mathrm{C}\right)$ and $(\mathrm{c})$ and $(\mathrm{d})$ hyperthermic $\left(40\right.$ or $\left.42^{\circ} \mathrm{C}\right)$ preconditioned hearts subjected to $40 \mathrm{~min}$ of ischemia; (e) normal or (f) preconditioned hearts perfused with $17 \beta$-estradiol and exposed to $40 \mathrm{~min}$ of ischemia; (g) normal or $(\mathrm{h})$ preconditioned hearts derived from $17 \beta$-estradiol-pretreated rats, exposed to $40 \mathrm{~min}$ of ischemia. Firstminute reperfusion samples of coronary effluent were collected for estimation of TNF- $\alpha$ after ischemia. Results: These studies show significant myocardial and coronary vascular depressant effects of TNF- $\alpha: P_{\max }$ and CF were reduced, whereas left ventricular enddiastolic pressure (LVEDP) and coronary vascular resistance $(\mathrm{CVR})$ were increased. Hyperthermic $\left(42^{\circ} \mathrm{C}\right)$ preconditioning offered better protection against TNF- $\alpha$ compared to normothermic preconditioning. $17 \beta$-Estradiol and hyperthermic preconditioning $\left(42^{\circ} \mathrm{C}\right)$ normalized LVEDP, $\mathrm{CF}$ and $\mathrm{CVR}$ but not $\mathrm{P}_{\max }$ against depressant effects of TNF- $\alpha$. The most significant benefit of combining $17 \beta$-estradiol with hyperthermic preconditioning was the normalization of $\mathrm{P}_{\max }$. Postischemic recovery in $\mathrm{P}_{\max }$ and CF was significantly reduced, unlike postischemic recovery in LVEDP and CVR that was increased. Hyperthermic $\left(42^{\circ} \mathrm{C}\right)$ preconditioning improved recovery of $\mathrm{P}_{\max }$ and LVEDP and normalized CF and $17 \beta-$ estradiol completely normalized $\mathrm{P}_{\max }$ and LVEDP but had no effect on recovery of $\mathrm{CF}$ and CVR. No additive benefit was offered when $17 \beta$-estradiol was combined with hyperthermic preconditioning. Myocardial production of $\mathrm{TNF}-\alpha$ was reduced by hyperthermic ischemic preconditioning and by $17 \beta$-estradiol treatment. Conclusions: (a) TNF- $\alpha$ exerts direct myocardial and coronary vascular depressant effects that can be reversed by hyperthermic $\left(42^{\circ} \mathrm{C}\right)$ preconditioning and by $17 \beta$-estradiol treatment; (b) unprotected I/R caused severe derangement of left ventricular functions accompanied by increased myocardial production of TNF- $\alpha$; (c) hyperthermic $\left(42^{\circ} \mathrm{C}\right)$ preconditioning and $17 \beta$-estradiol treatment provide significant protection against I/R injury; (d) hyperthermic $\left(42^{\circ} \mathrm{C}\right)$ preconditioning when combined with $17 \beta$-estradiol treatment provides additive protection only to $\mathrm{P}_{\max }$ after $\mathrm{TNF}-\alpha$; (e) increased myocardial production of TNF- $\alpha$ contributes to I/R injury.

Prof. Jasbir Singh Juggi (supervisor)

Prof. A.S. Mustafa (co-supervisor) 


\section{Abstracts of Theses Approved for PhD Degree at the Faculty of Medicine, Health Sciences Centre, Kuwait University}

\author{
14 \\ Development of a Chimaeric \\ Anticarcinoembryonic Antigen (CEA) Antibody \\ by Gene Targeting \\ Abir A. El-Abdallah \\ Department of Microbiology, Faculty of Medicine, \\ Kuwait University
}

Targeted immunotherapy using monoclonal antibodies has gained much importance in cancer management. There are currently 5 monoclonal antibodies that are FDA approved and in use in clinics in cancer treatment; none of these are targeted to colorectal cancer. 11-285-14 is a mouse hybridoma that secretes an anticarcinoembryonic antigen monoclonal antibody which has shown promising therapeutic effects in targeting colorectal cancer cells in vitro and in vivo. To take this antibody further into clinical application, a less immunogenic version was needed. The objective of this study was to develop a chimaeric version of 11-285-14 using a gene-targeting approach. Two replacement vectors were constructed to insert the human constant $\gamma 1$ gene within the mouse heavy chain locus of 11-285-14 hybridoma cells. The mouse constant $\gamma 1$ gene $(1.5 \mathrm{~kb})$ and the mouse $\mu$ intron fragment $(2.2 \mathrm{~kb})$ were amplified by PCR and cloned into a pKO scrambler vector. The human constant $\gamma 1$ gene fragment $(2.2 \mathrm{~kb})$ was cloned next to the intron fragment. The 11-285-14 VDJ region was cloned in frame to the human constant gene in one of the vectors. The two vectors were electroplated into hybridoma cells. Resistant colonies were screened by ELISA. Of the 4,370 resistant colonies obtained, 87 colonies showed discretion of the human isotype at a level between $4 \mathrm{ng}$ and $32 \mathrm{ng} / \mathrm{ml}$. PCR and Southern blot results confirmed the correct integration of the human gene by replacement events within the heavy chain locus. Most of the producers ceased to express the human isotype within a few weeks after the initial positive ELISA results. Instability of secretion was not only due to the loss of the human gene but to other undefined epigenetic or physiologic reasons.

Prof. C.H.J. Ford (supervisor)

\section{5 \\ Early Glomerular Changes in Diabetic Growth Hormone-Deficient Rats}

Slava Adel Malatiali

Department of Physiology, Faculty of Medicine, Kuwait University

Introduction: This study explores the development of early glomerular hypertrophy and hyperfiltration in diabetic growth hormone-deficient (dwarf) rats, describes the sequence of glomerular morphological and early hemodynamic changes that occur, the reversibility of these changes on treatments with insulin or phlorizin (PLZ) and their correlations with proteinuria. Methods: Diabetes was induced in 8-week-old male dwarf rats with streptozotocin. Morphometric studies were performed on kidney sections stained with PAS, or with cytoplasmic (anti-ezrin) or nuclear (anti-Wilm's tumor1) podocyte antibodies. Clearances of para-aminohippurate $\left(\mathrm{C}_{\mathrm{PAH}}\right)$, inulin $\left(\mathrm{C}_{\text {inulin }}\right)$ and renal blood flow were measured. Results: At 1week diabetes, glomerular tuft area increased by $9.5 \%$, with a $54 \%$ increase in the ezrin-positive (podocyte) area and no change in the PAS-positive mesangial matrix (MMA). Podocyte density decreased with growth of the tuft and unchanged podocyte number. At 8 weeks of diabetes, tuft area grew by $28.5 \%$ with increment in MMA $(115.9 \%)$ and no further podocyte hypertrophy. Podocyte density decreased further despite a $12 \%$ increase in podocyte number. Oneweek treatment with PLZ, but not with insulin, prevented the podocyte changes. At 1-week diabetes, $\mathrm{C}_{\mathrm{PAH}}$ and $\mathrm{C}_{\text {inulin }}$ increased by $80 \%$ indicating hyperperfusion and hyperfiltration, respectively, with no change in their ratio. Normalization of glycemia with insulin or PLZ prevented these hemodynamic changes. Diabetes caused a 2- to 3fold increase in protein excretion rate, which was also prevented by 1-week PLZ or insulin treatments. Proteinuria was highly correlated with $C_{\text {inulin }}\left(r^{2}=0.6\right)$ and less so with podocyte changes $\left(r^{2}=0.3\right)$. Conclusions: Growth hormone is not required for development of early diabetic glomerular hypertrophy and hyperfiltration. Early in diabetes, podocyte changes precede MMA expansion and are reduced by short-term normalization of the glycemia with PLZ but not with insulin, with which glycemia was more variable. Hyperfiltration and hyperperfusion detected early in diabetes are due to preglomerular vasodilatation and are both prevented by normalization of glycemia, even in the absence of insulin. Thus, hyperglycemia appears to be the major determinant of these changes and of proteinuria, which in early diabetes is strongly associated with glomerular hyperfiltration and weakly associated with podocyte changes.

Prof. Mario Barac-Nieto (supervisor)

Dr. I. Francis (co-supervisor)

Dr. C. Cojocel (co-supervisor) 